\title{
Caso 5/2006 - Choque em Mulher de 78 Anos Portadora de Cardiomiopatia Hipertrófica e Fibrilação Atrial Persistente
}

\author{
Case 5/2006 - Shock in a 78 Year-old Woman with Hypertrophic \\ Cardiomyopathy and Persistent Atrial Fibrillation
}

Edmundo Arteaga e Paulo Sampaio Gutierrez Instituto do Coração do Hospital das Clínicas - FMUSP - São Paulo, SP

Mulher de 78 anos de idade, portadora de cardiomiopatia hipertrófica, procurou atendimento médico no Hospital em razão de dor torácica irradiada para o dorso, acompanhada de choque cardiogênico.

A paciente foi informada do diagnóstico de cardiopatia há 17 anos (1986). Nessa época, então com 61 anos de idade, havia sido submetida a cinecoronariografia a qual revelou coronárias sem lesões obstrutivas e microfístulas em território da artéria coronária esquerda. A ventriculografia esquerda foi considerada normal.

A paciente foi atendida pela primeira vez neste Hospital há 14 anos (1989), depois de internação em outro Serviço em razão de dor precordial e arritmia cardíaca. Recebeu alta dessa internação com prescrição de digoxina $0,25 \mathrm{mg}$ e sulfato de quinidina $600 \mathrm{mg}$.

O exame físico (set/1989) revelou peso 68 kg; altura 1,65 m, freqüência cardíaca 72 bpm. Foi diagnosticado aumento difuso do volume da tireóide. O restante do exame físico não demonstrou anormalidades.

O eletrocardiograma (ECG) (set/1989) revelou fibrilação atrial, freqüência cardíaca 90 bpm, sobrecarga ventricular esquerda (índice de Sokolow de $60 \mathrm{~mm}$ ) e alterações da repolarização ventricular com ondas $T$ negativas em derivações precordiais esquerdas. (fig. 1).

A creatinina sérica foi $0,8 \mathrm{mg} / \mathrm{dl}$, o sódio $142 \mathrm{mEq} / \mathrm{l}$, o potássio 4,2 mEq/l, a concentração de TSH <0,1 $\mu \mathrm{Ul} / \mathrm{ml}$ e de tiroxina $14 \mu \mathrm{g} / \mathrm{dl}$.

As medicações em uso foram substituídas por disopiramida $450 \mathrm{mg}$, depois substituída por verapamil $240 \mathrm{mg} / \mathrm{dia}$

O ECG dinâmico (dez/1989) revelou ritmo básico sinusal, extra-sístoles atriais $<5 /$ h, várias taquicardias atriais de 5 a 6 batimentos, extra-sístoles ventriculares 10/h, um episódio de taquicardia ventricular de 19 batimentos.

O teste ergométrico (9/11/90) realizado em ritmo de fibrilação atrial demonstrou freqüência cardíaca em repouso 95 bpm, freqüência cardíaca no esforço máximo 159 bpm, pressão arterial em repouso 109/81 mmHg e no esforço máximo $156 / 64 \mathrm{mmHg}$. A duração total do teste foi 10 min $15 \mathrm{~s}$. 0 teste foi interpretado como sem evidência de isquemia.

O ECO (1990) revelou dilatação de átrio esquerdo e função sistólica de ventrículo esquerdo normal, sem alterações valvares (tab. 1). Na evolução, ecocardiogramas foram realizados e revelaram hipertrofia de ventrículo com relação septo/parede posterior $>1,5$ e grande dilatação de átrio esquerdo. Além disso, foi também diagnosticada na evolução hipertensão progressiva de ventrículo direito (tab. 1)

A paciente evoluiu por 11 anos com queixas de palpitações paroxísticas e dispnéia desencadeada por médios esforços. Nesse ínterim foi acrescentado o tratamento com varfarina. O INR foi mantido entre 1,5 e 2,8 .

A partir de setembro de 2000 iniciou dispnéia aos pequenos esforços e repouso, e, em alguns meses, franca ortopnéia necessitando internação para alívio dos sintomas. Nessa época fazia uso de $25 \mathrm{mg}$ de atenolol, $40 \mathrm{mg}$ de enalapril, $25 \mathrm{mg}$ de hidroclorotiazida, $40 \mathrm{mg}$ de furosemida, $25 \mathrm{mg}$ de espironolactona e 2,5 $\mathrm{mg}$ de varfarina.

Os exames laboratoriais (mar/2003) revelaram 11,9 g/ dl de hemoglobina, hematócrito 37\%, glicemia $99 \mathrm{mg} / \mathrm{dl}$, colesterolemia 122 mg/dl (46 mg/dl de HDL-colesterol e $67 \mathrm{mg}$ de LDL-colesterol) e $45 \mathrm{mg} / \mathrm{dl}$ de triglicérides.

A paciente foi trazida ao hospital por dor interescapular aguda, dispnéia e edema de membros inferiores, além de vômitos. O exame físico (14/8/2003) revelou paciente em mau estado geral, pressão arterial 120/70 mmHg, freqüência cardíaca $110 \mathrm{bpm}$. A semiologia pulmonar revelou estertores difusos. 0 exame do coração revelou ritmo cardíaco irregular, sopro sistólico $+++/ 4$ em área mitral. Ao exame do abdome o fígado foi palpado a 4 $\mathrm{cm}$ da borda costal. Havia edema discreto de membros inferiores.

Editor da Seção: Alfredo José Mansur (ajmansur@incor.usp.br)

Editores Associados: Desidério Favarato (dclfavarato@incor.usp.br)

Vera Demarchi Aiello (anpvera@incor.usp.br)

Correspondência: Edmundo Arteaga • InCor - Av. Dr. Enéas C. Aguiar, 44-05403-000 - São Paulo, SP

Email: edmundo.arteaga@incor.usp.br 


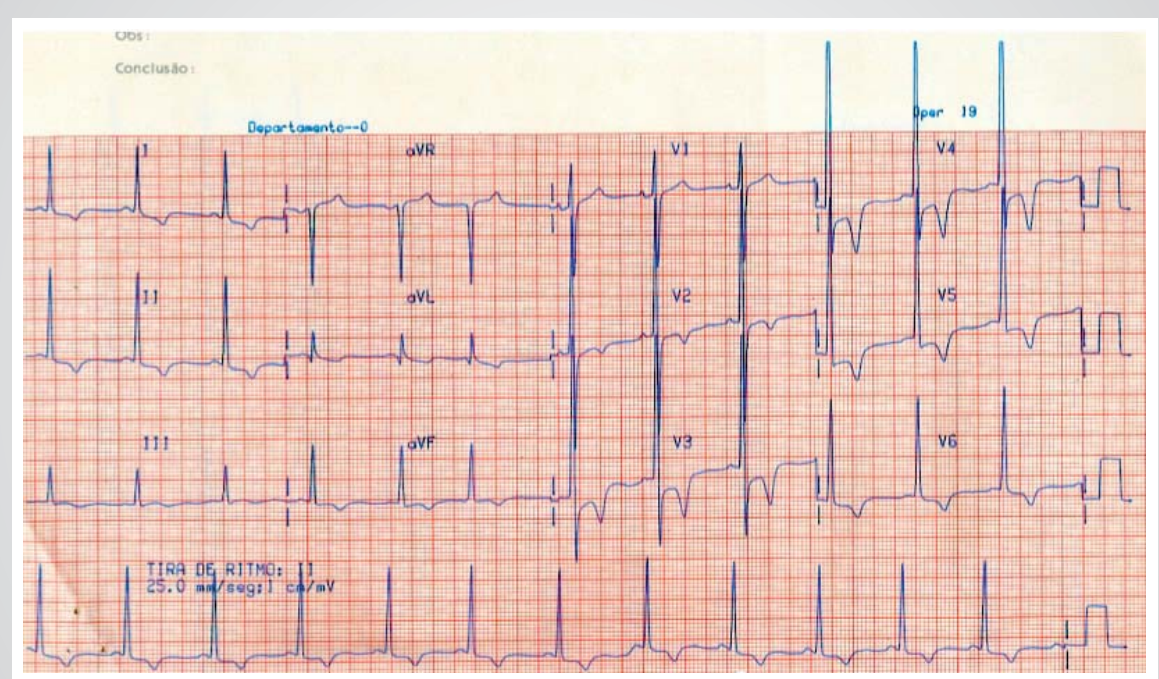

Fig. 1 - Eletrocardiograma. Ritmo de fibrilação atrial e intensa sobrecarga ventricular esquerda, com alterações secundárias da repolarização ventricular.

\begin{tabular}{|c|c|c|c|c|c|c|c|}
\hline & & & & & & & 2003 \\
\hline Ritmo & FA & FA & FA & FA & FA & FA & FA \\
\hline Septo $(m m)$ & 10 & 18 & 21 & 14 & 16 & 17 & 16 \\
\hline Parede posterior VE (mm) & 9 & 12 & 12 & 14 & 14 & - & 13 \\
\hline Diâmetro diastólico do VE (mm) & 48 & 48 & 45 & 50 & 56 & 58 & 58 \\
\hline Diâmetro sistólico do VE (mm) & - & & 27 & 35 & 41 & 40 & - \\
\hline Aorta (mm) & 33 & 34 & 28 & 31 & 32 & - & 30 \\
\hline Átrio esquerdo (mm) & 51 & 51 & 56 & 60 & 74 & 77 & 70 \\
\hline Pressão sistólica VD (mm Hg) & - & - & 30 & 34 & 60 & 120 & 75 \\
\hline Fração de ejeção do VE (\%) & 73 & 82 & 78 & 65 & 60 & 67 & 60 \\
\hline Padrão de fluxo transvalvar mitral & $E<A$ & $E<A$ & $E<A$ & - & - & - & - \\
\hline Insufifiência mitral & Discreta & Discreta & Discreta & Discreta & Acentuada & Moderada & Acentuada \\
\hline Insuficiência tricúspide & Leve & Leve & Moderada & moderada & Acentuada & Acentuada & Acentuada \\
\hline
\end{tabular}

O ECG (ago/2003) mostrou fibrilação atrial, frequêencia cardíaca 110 bpm, sobrecarga atrial direita (PeñalozaTranchesi) e alterações da repolarização ventricular (fig. 2).

A paciente sofreu parada cardíaca em assistolia algumas horas após a internação. Foi reanimada e evoluiu com choque, cianose intensa, oligúria e hipertermia.

Os exames laboratoriais revelaram acidose metabólica, insuficiência renal (tab. 2). A urocultura foi positiva para Escherichia coli. A radiografia de tórax no segundo dia de internação foi considerada sugestiva de broncopneumonia e foi iniciado o tratamento com ceftriaxona. Apesar de uso de doses crescentes de dopamina e depois noradrenalina, a paciente permaneceu hipotensa e faleceu na manhã de 19/8/2003.

\section{Aspectos clínicos}

Trata-se de uma paciente de 78 anos de idade com diagnóstico de cardiomiopatia hipertrófica $(\mathrm{CH})$ forma não obstrutiva, com longa evolução (17 anos). O diagnóstico da doença é feito pelos sintomas: dispnéia, palpitações, dor precordial (referidos pela paciente), e síncope; e pelos exames complementares: o ECG mostrava ritmo de fibrilação atrial e sobrecarga ventricular esquerda; ao ecocardiograma observou-se hipertrofia do miocárdio $>15$ mm.; e no estudo de eletrocardiografia dinâmica taquicardia ventricular não sustentada.

Chamou-nos a atenção o início tardio dos sintomas (61 anos de idade), uma vez que, geralmente estes costumam aparecer na terceira ou quarta década de vida e a experiência mostra que pacientes que atingem a sexta década dificilmente apresentam sintomas e têm uma sobrevida semelhante à da população normal nessa faixa etária ${ }^{1,2}$.

Desde a primeira avaliação no Incor (1990) a paciente já apresentava fibrilação atrial ao ECG, aumento do átrio esquerdo e sinais de disfunção diastólica ao ECO o que é característico da doença, assim como arritmia ventricular complexa ao Holter. Apesar de ter sido medicada com 


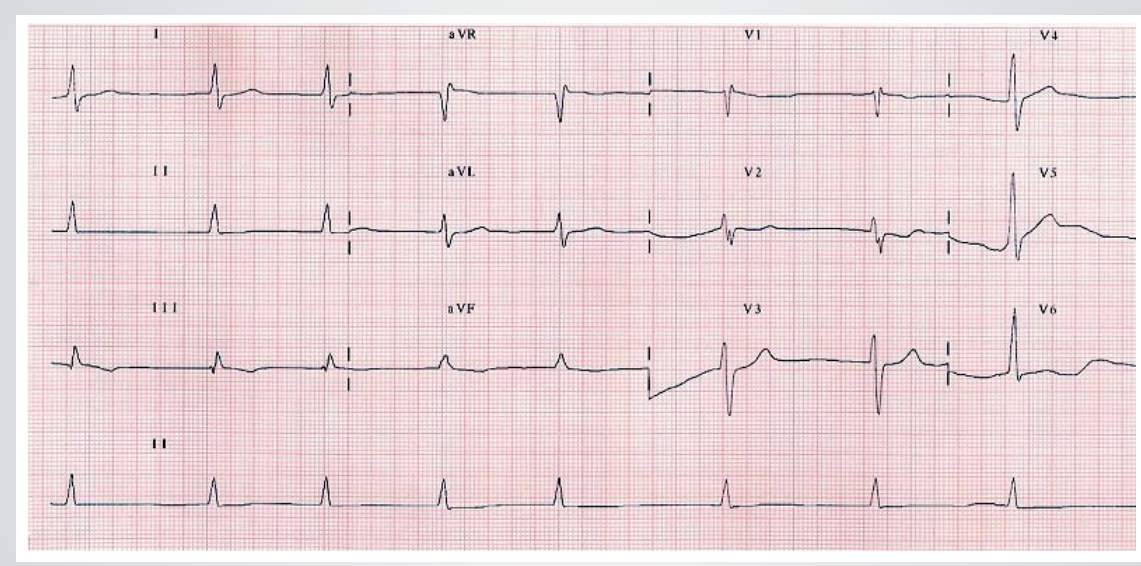

Fig. 2 - Eletrocardiograma. Ritmo de fibrilação atrial, houve diminuição da amplitude dos complexos QRS em relação ao ECG inicial (fig. 1).

\begin{tabular}{|c|c|c|c|}
\hline & 14 ago & 15 ago & 18 ago \\
\hline Hemoglobina (g/dl) & 12,9 & 13,2 & 14,4 \\
\hline Hematócrito (\%) & 39 & 42 & 43 \\
\hline Leucócitos $/ \mathrm{mm}^{3}$ & 4700 & 8214 & 12200 \\
\hline Neutrófilos (\%) & 65 & 77 & 78 \\
\hline Linfócitos (\%) & 25 & 14 & 13 \\
\hline Monócitos (\%) & 10 & 9 & 9 \\
\hline Uréia (mg/dl) & 139 & 149 & 159 \\
\hline Creatinina (mg/dl) & 1,8 & 2,8 & 2,5 \\
\hline Sódio (mEq/l) & - & 138 & 136 \\
\hline Potássio (mEq/l) & - & 4,5 & 6 \\
\hline Glicose (mEq/l) & 77 & 20 & 98 \\
\hline Gasimetria & $\mathrm{FlO}_{2} 60 \%$ & & $\mathrm{FIO}_{2} 40 \%$ \\
\hline $\mathrm{pH}$ & 7,27 & & \\
\hline $\mathrm{pCO}_{2}(\mathrm{~mm} \mathrm{Hg})$ & 36 & & \\
\hline $\mathrm{pO}_{2}(\mathrm{~mm} \mathrm{Hg})$ & 147 & & \\
\hline Sat $\mathrm{O}_{2}(\%)$ & 78,7 & & 99 \\
\hline $\mathrm{HCO}_{3}(\mathrm{mEq} / \mathrm{l})$ & 16 & & \\
\hline $\mathrm{EB}(\mathrm{mEq} / \mathrm{l})$ & $-9,8$ & & \\
\hline Proteinas totais ( $\mathrm{g} / \mathrm{dl}$ ) & & & 3,4 \\
\hline Albumina (g/dl) & & & 1,5 \\
\hline
\end{tabular}

Disopiramida, Verapamil e Atenolol, a paciente teve pouca melhora; porém se manteve estável até o ano 2000, quando apresentou piora significativa dos sintomas. Paralelamente houve piora significativa da anatomia e função cardíaca, demonstrada pela ecocardiografia, com aumento do átrio $(60 \mathrm{~mm}$ para $70 \mathrm{~mm}$ ) e ventrículo esquerdo (50 $\mathrm{mm}$ para $58 \mathrm{~mm}$ ), aumento da pressão sistólica do ventrículo direito, e aumento do grau de insuficiência mitral e tricúspide; porém com função sistólica preservada.

Apesar da paciente ter recebido medicação para disfunção sistólica, teve evolução desfavorável e progressiva até sua última internação, em quadro clínico de choque cardiogênico, e certamente disfunção sistólica e diastólica. Esse tipo de evolução para dilatação ventricular esquerda com disfunção sistólica e diastólica ocorre em $10 \%$ dos pacientes na fase avançada da doença como neste caso ${ }^{1}$.

Cabe discutir se haveria algum outro tipo de tratamento que pudesse ter melhorado a qualidade de vida da paciente. Em se tratando de paciente com diagnóstico de cardiomiopatia hipertrófica forma não obstrutiva, o tratamento invasivo com marcapasso, oclusão do ramo septal da artéria coronária descendente anterior pelo álcool e cardiomiectomia transvalvular aórtica não estão indicados ${ }^{3,4}$.

Com base em trabalhos ${ }^{5}$ que demonstraram que bloqueadores de receptores da angiotensina II, diminuem a ação trófica e pró-fibrótica da mesma, realizamos um trabalho utilizando o Losartan $100 \mathrm{mg} /$ dia durante 6 meses em pacientes com disfunção diastólica e não obstrutivos, e observamos melhora da disfunção diastólica avaliada pela ecocardiografia, da classe funcional, e da fração terminal do BNP (NT pró-BNP) ${ }^{6,7}$.

Em relação aos fatores preditores de morte súbita como: parada cardiorrespiratória recuperada, taquicardia ventricular sustentada, síncope recorrente, taquicardia ventricular não sustentada, genótipo de risco ou morte súbita em 2 ou mais parentes de $1^{\circ}$ grau com menos de 45 anos, jovens menores de 20 anos, hipertrofia ventricular acima de $30 \mathrm{~mm}$ principalmente em jovens, resposta hipotensora ao exercício, gradiente obstrutivo elevado e evolução para forma dilatada ${ }^{3,8}$; a paciente tinha apenas arritmia complexa ao Holter, cuja prevalência aumenta com o avanço da idade, sendo a morte súbita é mais freqüente em pacientes jovens ${ }^{1,3}$.

(Dr. Edmundo Arteaga)

\section{Hipóteses diagnósticas}

Cardiomiopatia hipertrófica, evolução final com dilatação.

\section{NeCropsia}

À necropsia, havia grande hipertrofia biventricular cardíaca simétrica (fig. 3), compatível quer com miocardiopatia hipertrófica simétrica quer com alteração secundária a hipertensão arterial sistêmica. Com o estudo microscópico, pôde-se estabelecer o diagnóstico 
de miocardiopatia, pois havia desarranjo significativo de fibras miocárdicas (fig. 4).

As artérias coronárias mostravam grande calibre e tortuosidades (fig. 5), mas não obstruções. É possível que se trate apenas de variação anatômica, uma vez que não havia lesões isquêmicas do miocárdio, a despeito da hipertrofia. Porém, cateterismo antigo evidenciou microfístulas, cujas comprovação e importância fisiopatológica são difíceis de estabelecer.

A paciente apresentou tromboembolismo pulmonar (fig. 6), que, levando a baixo débito cardíaco, causou isquemia mesentérica e foi o principal responsável pela morte.

A paciente tinha também hipertireoidismo. Embora as alterações anátomopatológicas sejam mais sugestivas de bócio adenomatoso, este usualmente não causa o distúrbio funcional apresentado pela paciente. Assim, é possível que a lesão inicial fôsse bócio tóxico (doença de Graves), porém com modificações introduzidas pela medicação.

Finalmente, houve suspeita clínica de quadro infeccioso no período terminal. Não houve comprovação morfológica disso, talvez por cura decorrente de tratamento antimicrobiano adequado.

\section{(Dr. Paulo Sampaio Gutierrez)}

\section{Hipóteses diagnósticas}

Doença principal: miocardiopatia hipertrófica. “Causa mortis": isquemia mesentérica após tromboembolismo pulmonar.

\section{(Dr. Paulo Sampaio Gutierrez)}

\section{Potencial Conflito de Interesses}

Declaro não haver conflitos de interesses pertinentes.

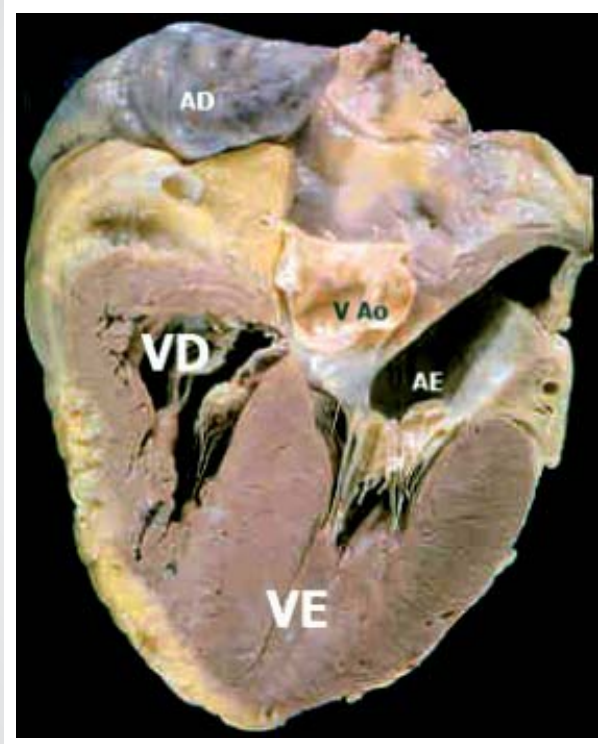

Fig. 3 - Corte frontal do coração, mostrando acentuada hipertrofia concêntrica da parede do ventrículo esquerdo. AD- Átrio direito (apêndice auricular); $A E$ - Átrio esquerdo; V Ao-valva aórtica; VD-ventrículo direito; VE-ventrículo esquerdo.

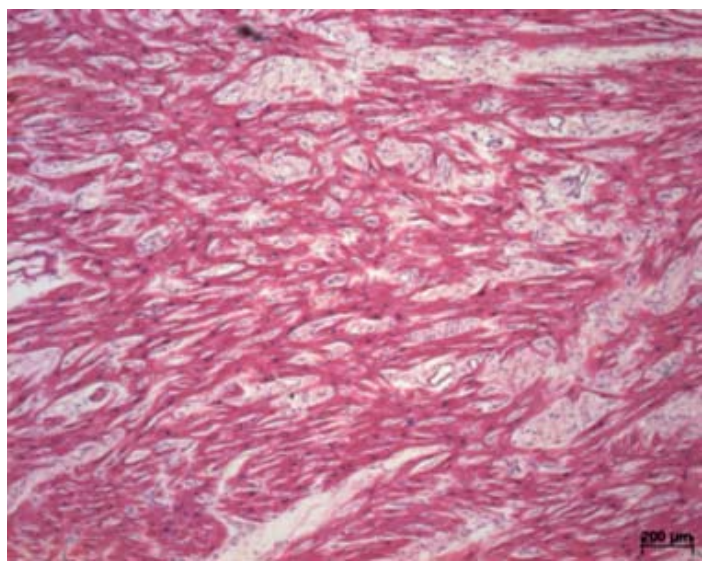

Fig. 4 - Corte histológico do miocárdio, mostrando desarranjo das fibras miocárdicas. Coloração pela hematoxilina e eosina, aumento da objetiva 5x. 


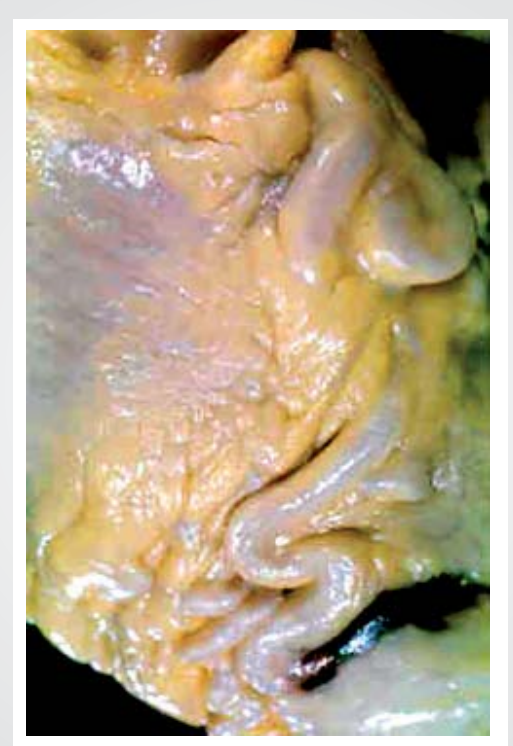

Fig. 5 - Visão externa do ramo interventricular anterior (descendente anterior) da artéria coronária esquerda, mostrando tortuosidade e dilatação.

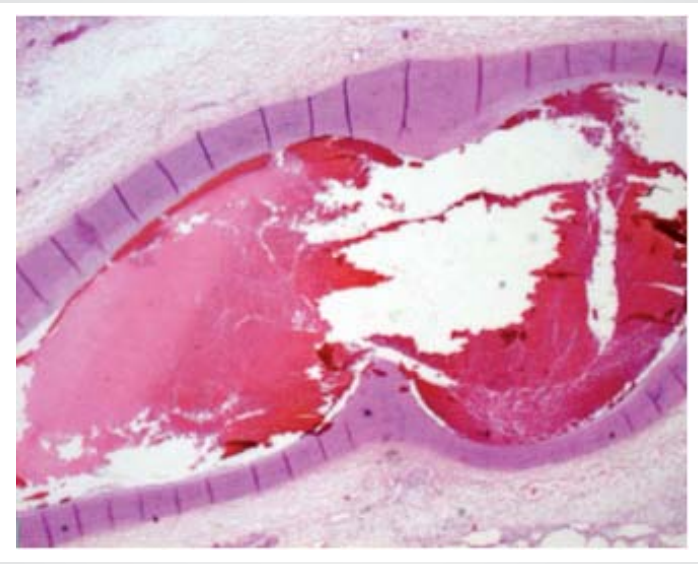

Fig. 6 - Corte histológico de pulmão. Vê-se obstrução tromboembólica de ramo da artéria pulmonar. Coloração pela hematoxilina e eosina, aumento da objetiva $2,5 x$.

\section{REFERÊNCIAS}

1. Maron BJ. Hypertrophic cardiomyopathy. A systematic review. JAMA 2002;287:1308-20.

2. Arteaga E, lanni BM, Fernandes F, Mady C. Benign outcome in a longterm follow-up of patients with hypertrophic cardiomyopathy in Brazil. Am Heart J 2005;149:1099-105.

3. Maron BJ, McKenna WJ, Danielson GK, Kappenberger LJ, Kuhn HJ, Seidman CE, et al; Task Force on Clinical Expert Consensus Documente American College of Cardiology; Committee for Practice Guidelines. European Society of Cardiology. J Am Coll Cardiol 2003;42:1687713.

4. Arteaga E, Araujo AQ. Cardiomiopatia hipertrófica: tratamento invasivo e não invasivo. Rev Soc Cardiol Estado de São Paulo 2003;4:499508.
5. Lim DS, Lutucuta S, Bachireddy P, Youker K, Evans A, Entman M, et al. Angiotensin II blockade reverses myocardial fibrosis in a transgenic mouse model of human hypertrophic cardiomyopathy. Circulation 2001;103:789-91.

6. Araujo $A Q$, Arteaga E, lanni BM, Buck PC, Rabello R, Mady C. Effect or Losartan on left ventricular diastolic function in patients with nonobstructive hypertrophic cardiomyopathy. Am J Cardiol 2005;96:1563-1567.

7. Arteaga E, Araujo AQ, Buck P, lanni BM, Rabello R, Mady C. Plasma amino-terminal pro-B-type natriuretic peptide quantification in hypertrophic cardiomyopathy. Am Heart J 2005;150:1228-32.

8. Elliott PM, Poloniecki J, Dickie S, Sharma S, Monserrat L, Varnava A et al. Sudden death in hypertrophic cardiomyopathy: identification of high risk patients. J Am Coll Cardiol 2000;36:2212-8. 